\title{
EXAMPLES OF BUCHSBAUM QUASI-GORENSTEIN RINGS
}

\author{
MANFRED HERRMANN AND NGGÔ VIÊT TRUNG \\ (Communicated by Louis J. Rafliff, Jr.)
}

\begin{abstract}
The paper shows the existence of Buchsbaum quasi-Gorenstein rings of any admissible depth.
\end{abstract}

\section{INTRODUCTION}

Until now few examples of non-Cohen-Macaulay prime almost complete intersections were known. One possibility to find such examples is to go through linkages. According to an idea of Peskine and Szpiro [6], every prime ideal linked to a quasi-Gorenstein ideal is an almost complete intersection, cf. [9]. Recall that an ideal $I$ of a ring $A$ is called quasi-Gorenstein if the factor ring $A / I$ is quasi-Gorenstein, i.e., the canonical module of $A / I$ is isomorphic to $A / I$. However, to find non-Cohen-Macaulay quasi-Gorenstein ideals is usually also hard. For instance, Schenzel [8] used a result of Mumford on abelian varieties to give a class of non-Cohen-Macaulay quasi-Gorenstein rings that are Buchsbaum with depth 2 .

The aim of this paper is to construct Buchsbaum quasi-Gorenstein rings of any admissible depth that are generated by monomials. Note that from the description of the local cohomology of the canonical module of a Buchsbaum ring [8] once can easily deduce that the depth $t$ of a Buchsbaum quasiGorenstein ring $A$ is either $\operatorname{dim} A$, i.e., $A$ is a Cohen-Macaulay ring, or $2 \leq t \leq[(\operatorname{dim} A+1) / 2]$. Rings generated by monomials are, in other terms, affine semigroup rings whose structures can be described well by means of the underlying affine semigroups $[11,7]$. For instance, there exist sufficient (and necessary) conditions for such rings to be Cohen-Macaulay, Gorenstein, or Buchsbaum. Using the theory of affine semigroup rings, we can translate the problem of constructing quasi-Gorenstein rings generated by monomials to one of finding certain kinds of systems of diophantine homogeneous linear equations. From this we then derive examples of (non-Cohen-Macaulay) Buchsbaum quasiGorenstein rings and, therefore, of Buchsbaum almost complete intersection rings of any admissible depth. Compared with Schenzel's results, our method

Received by the editors January 4, 1991 and, in revised form, June 27, 1991.

1991 Mathematics Subject Classification. Primary 13H10.

The second author was supported by the Alexander von Humboldt Foundation.

(C) 1993 American Mathematical Society $0002-9939 / 93 \$ 1.00+\$ .25$ per page 
has the advantage of being more explicit: one knows the parametric presentation of the given quasi-Gorenstein rings, and therefore one can compute their defining equations.

This paper was written while the second author was visiting the University of Essen and the University of Cologne by a grant of the Alexander von Humboldt Foundation. He would like to thank these institutions for their support and hospitality.

\section{AfFINE SEMIGROUP RINGS}

In this section we collect some results on affine semigroup rings that will be used in the construction of Buchsbaum quasi-Gorenstein rings.

Let $\mathbb{N}$ denote the set of nonnegative integers. By an affine semigroup we mean a finitely generated submonoid $S$ of the additive monoid $\mathbb{N}^{n}, n>0$. Let $k[S]$ denote the semigroup ring of $S$ over a field $k$. Then one can identify $k[S]$ with the subring of a polynomial ring $k\left[t_{1}, \ldots, t_{n}\right]$ generated over $k$ by the monomials $t^{x}=t_{1}^{x_{1}}, \ldots, t_{n}^{x_{n}}, x=\left(x_{1}, \ldots, x_{n}\right) \in S$. Of course, every ring generated over $k$ by a finite set of monomials is an affine semigroup ring.

For further investigations we have to introduce some notation. Let $A$ and $B$ be two subsets of $\mathbb{Z}^{n}$. We denote by $G(A)$ the additive subgroup of $\mathbb{Z}^{n}$ generated by $A$, and by $A \pm B$ the set of all elements $a \pm b, a \in A$ and $b \in B$. Moreover, we denote by $k[A]$ the $k$-vector subspace of $k[G(A)]$ spanned by the elements of $A$. If $A+S \subseteq A$, we call $A$ an $S$-ideal. In this case, $k[A]$ has a natural $\mathbb{Z}^{n}$-graded module structure over $k[S]$. If $A=B \backslash C$ for $S$-ideals $B \supseteq C$, we will identify $k[A]$ with the factor module $k[B] / k[C]$.

Let $S$ be an arbitrary affine semigroup in $\mathbb{N}^{n}$ with $\operatorname{rank}_{Z} G(S)=r \geq 1$. Let $\mathscr{C}_{S}$ denote the convex polyhedral cone spanned by the elements of $\bar{S}$ in the space $\mathbb{Q}^{n}$. Then we call $S$ a standard affine semigroup if $\mathscr{C}_{S}$ has exactly $n(r-1)$-dimensional faces lying on the hyperplanes $x_{i}=0, i=1, \ldots, n$. According to Hochster [4, p. 323] (see also [11, §1]), every affine semigroup can be transformed isomorphically to a standard one.

Set $S_{(i)}=\left\{x \in S \mid x_{i}=0\right\}$ and $S_{i}=S-S_{(i)}, i=1, \ldots, n$. Note that the sets $S_{i}$ are $S$-ideals. Put

$$
C_{S}=G(S) \backslash \bigcup_{i=1}^{n} S_{i}
$$

Let $H_{M}^{i}(k[S])$ denote the $i$ th local cohomology module of $k[S]$ with respect to the maximal ideal $M=k[S \backslash(0)], i=0, \ldots, r$. Note that $\operatorname{dim} k[S]=r$. From the fact that $\operatorname{Hom}_{k}\left(H_{M}^{r}(k[S]), k\right)$ is the canonical module of $k[S]$, we derive the following criterion for $k[S]_{M}$ to be a quasi-Gorenstein ring (which is implicitly contained in $[2,11])$.

Lemma 1.1. Let $S$ be a standard affine semigroup. Then $k[S]_{M}$ is a quasiGorenstein ring if and only if there exists an element $y \in G(S)$ such that $C_{s}=$ $y-S$.

Proof. Obviously $k\left[C_{S}\right]$ has the structure of a factor module over $k[S]$. By [11, Corollary 3.8] we have $H_{M}^{r}(k[S]) \cong k\left[C_{S}\right]$. It is easily seen that

$$
\operatorname{Hom}_{k}\left(k\left[C_{S}\right], k\right) \cong k\left[-C_{S}\right] \text {. }
$$


But $k\left[-C_{S}\right] \cong k[S]$ if and only if there exists an element $y \in G(S)$ (which corresponds to the shifting degree of the isomorphism) such that $y-C_{S}=S$ or equivalently $C_{S}=y-S$.

Unfortunately, one has been unable to find necessary and sufficient conditions for $k[S]_{M}$ to be a Buchsbaum ring in terms of $S$. However, there is a formula for the computation of the local cohomology modules of $k[S]$, and from the $\mathbb{Z}^{n}$ graded structure of the local cohomology modules of $k[S]$ one can sometimes decide that $k[S]_{M}$ is a Buchsbaum ring.

Let $[1, n]$ be the set of the integers $1, \ldots, n$. For every subset $J$ of the set $[1, n]$ we set

$$
G_{J}=\bigcap_{i \notin J} S_{i} \backslash \bigcup_{j \in J} S_{j}
$$

and denote by $\pi_{J}$ the simplicial complex of all nonempty subsets $I$ of $J$ such that $\bigcap_{i \in I} S_{(i)} \neq\{0\}$. As usual, let $\widetilde{H}_{q}\left(\pi_{J}, k\right)$ denote the $q$ th reduced homology group of $\pi_{J}$ with coefficients in $k$.

Lemma 1.2 [11, Corollaries 3.4, 3.7]. Let $S$ be a standard affine semigroup such that $S=\bigcap_{i=1}^{n} S_{i}$. Then $H_{M}^{1}(k[S])=0$ and

$$
H_{M}^{i}(k[S]) \cong \bigoplus_{\substack{J \notin \pi_{11, n]} \\|J| \leq n-2}} k\left[G_{J}\right] \otimes_{k} \tilde{H}_{i-2}\left(\pi_{J} ; k\right)
$$

for all $i=2, \ldots, r-1$.

In the following we will denote by $E_{x}$ the $x$-graded part of a $\mathbb{Z}^{n}$-graded module over $k[S], x \in \mathbb{Z}^{n}$.

Lemma 1.3 [7, Theorem 6.10]. Set $V=\bigcup_{i=1}^{r-1}\left\{x \in \mathbb{Z}^{n} \mid\left[H_{M}^{i}(k[S])\right]_{x} \neq 0\right\}$. Suppose $x+y \notin V$ for all elements $x \in S \backslash(0)$ and $y \in V$. Then $k[S]_{M}$ is a Buchsbaum ring.

\section{BUCHSBAUM QUASI-GORENSTEIN RINGS OF ANY ADMISSIBLE DEPTH}

In this section we will apply the above criteria for quasi-Gorenstein and Buchsbaum affine semigroup rings to construct Buchsbaum quasi-Gorenstein rings of any admissible depth.

We will start with a subgroup $G$ of $\mathbb{Z}^{n}$ and try to impose conditions on $G$ so that there exists a submonoid $S$ of $\mathbb{N}^{n}$ with $G(S)=G$ for which $k[S]_{M}$ is a quasi-Gorenstein ring.

Theorem 2.1. Let $G$ be a subgroup of $\mathbb{Z}^{n}$ that satisfies the following conditions:

(i) For any $i=1, \ldots, n$ there is an element $x \in G$ with $x_{i}=0$ and $x_{j}>0$ for all $j \neq i$.

(ii) There exists an element $y \in G$ such that $y_{i}=1$ or -1 for all $i=$ $1, \ldots, n$.

Let $I$ be the set of the numbers $i$ with $y_{i}=1$ and set

$$
S=\left\{x \in G \cap \mathbb{N}^{n} \mid x_{i} \neq 1 \text { for all } i \in I\right\} .
$$

Then $k[S]_{M}$ is a quasi-Gorenstein ring.

Proof. We will first show that $S$ is a standard affine semigroup. Assumption (i) implies that there is an element $x \in S$ such that $x_{i}>0$ for all $i=1, \ldots, n$. 
Given any set of generators of $G$, one can add a sufficiently large multiple of $x$ to the generators to get a new set of generators of $G$ in $S$. From this it follows that $G(S)=G$. Let $L$ and $L_{i}$ denote the linear subspace of $\mathbb{Q}^{n}$ spanned by $G$ and $S_{(i)}$, respectively, $i=1, \ldots, n$. Let $H_{i}$ be the hyperplane $x_{i}=0$ of $\mathbb{Q}^{n}$. Since $L_{i}=L \cap H_{i}$ and $L+H_{i}=\mathbb{Q}^{n}$,

$$
\operatorname{dim}_{\mathbb{Q}} L_{i}=\operatorname{dim}_{\mathbb{Q}} L+\operatorname{dim}_{\mathbb{Q}} H_{i}-\operatorname{dim}_{\mathbb{Q}} \mathbb{Q}^{n}=\operatorname{dim}_{\mathbb{Q}} L-1 .
$$

From this it follows that the faces of $\mathscr{C}_{S}$ lying on $H_{i}$ have the maximal possible dimension. Moreover, assumption (i) also implies that $S_{(i)} \neq S_{(j)}$ for $i \neq j$. This means that these faces are different. Obviously, $\mathscr{C}_{S}$ has no other maximal faces. Hence $S$ is a standard affine semigroup. Further, from the definition of $S$ we deduce that

$$
S_{i}= \begin{cases}\left\{x \in G \mid x_{i} \geq 0 \text { and } x_{i} \neq 1\right\} & \text { if } i \in I, \\ \left\{x \in G \mid x_{i} \geq 0\right\} & \text { if } i \in I .\end{cases}
$$

Since $C_{S}=G \backslash \bigcup_{i=1}^{n} S_{i}$, we can compute $C_{S}$ and obtain

$$
C_{S}=\left\{x \in G \mid x_{i}<0 \text { or } x_{i}=1 \text { for } i \in I \text { and } x_{i}<0 \text { if } i \notin I\right\} \text {. }
$$

Now it is an easy matter to check that $C_{S}=y-S$. Thus, $k[S]_{M}$ is a quasiGorenstein ring by Lemma 1.1.

A subgroup of $\mathbb{Z}^{n}$ can be given as the set of all solutions in $\mathbb{Z}^{n}$ of a system of homogeneous linear equations in $n$ variables. Therefore, in order to construct quasi-Gorenstein rings we just need to find systems of homogeneous linear equations that have solutions $x$ with $x_{i}=0$ and $x_{j}>0, j \neq i$, for $i=1, \ldots, n$ and a solution $y$ with $y_{j}= \pm 1$.

Example (cf. [11, Example 4.2]). Let $G$ be the set of all solutions in $\mathbb{Z}^{n}$ of the equation $x_{1}+x_{2}=x_{3}+x_{4}$. This equation has the following solutions: $(0,2,1,1),(2,0,1,1),(1,1,0,2),(1,1,2,0),(1,-1,1,-1)$. Therefore, if we set

$$
S=\left\{x \in G \cap \mathbb{N}^{n} \mid x_{1} \text { and } x_{3} \neq 1\right\},
$$

then $A=k[S]_{M}$ is a quasi-Gorenstein ring with $\operatorname{dim} A=3$. By [11, Example 4.2], $A$ is a non-Cohen-Macaulay ring. Note that $S$ is isomorphic to the affine semigroup

$$
T=\left\{x \in \mathbb{N}^{3} \mid x_{1}+x_{2}-x_{3} \geq 0, x_{1} \neq 1, \quad x_{3} \neq 1\right\} .
$$

One can easily compute the generators of $T$ and obtain

$$
k[T]=k\left[t_{2}, t_{1}^{2}, t_{1}^{3}, t_{1}^{2} t_{3}^{2}, t_{2}^{2} t_{3}^{2}, t_{1}^{3} t_{3}^{2}, t_{1}^{3} t_{3}^{3}, t_{1}^{2} t_{2} t_{3}^{3}, t_{2}^{3} t_{3}^{3}, t_{1}^{4} t_{3}^{3}, t_{1}^{3} t_{2} t_{3}^{4}\right] .
$$

According to [8, Remark (3.2) and Theorem (3.3)], if $K_{A}$ is the canonical module of a Buchsbaum local ring $(A, \mathfrak{m})$, then $K_{A}$ satisfies Serre's condition $S_{2}$ and

$$
H_{\mathfrak{m}}^{i}\left(K_{A}\right) \cong H_{\mathrm{m}}^{d-i+1}(A)
$$

for all $i=2, \ldots, d-1, d=\operatorname{dim} A$. Moreover, if $A$ is a quasi-Gorenstein ring $\left(K_{A} \cong A\right)$, then $A$ is either a Cohen-Macaulay ring or $2 \leq \operatorname{depth} A \leq$ $[(d+1) / 2]$. In fact, the following result shows the existence of Buchsbaum quasi-Gorenstein rings of a given dimension $d$ of any admissible depth $t=$ $2, \ldots,[(d+1) / 2]$. 
Lemma 2.2. Let $G$ be the subgroup of the solutions in $\mathbb{Z}^{n}$ of the equation

$$
(t-1) X_{1}+X_{2}+\cdots+X_{t}=(n-t-1) X_{t+1}+X_{t+2}+\cdots+X_{n},
$$

where $t$ is an integer with $2 \leq t \leq[n / 2]$. Set

$$
S=\left\{x \in G \cap \mathbb{N}^{n} \mid x_{1} \neq 1, \quad x_{t+1} \neq 1\right\} .
$$

Then $A=k[S]_{M}$ is a Buchsbaum quasi-Gorenstein ring with $\operatorname{dim} A=n-$ 1 , depth $A=t$, and $H_{\mathfrak{m}}^{i}(A)=0$ for $i \neq t, n-t, n-1$. Moreover, $H_{\mathfrak{m}}^{t}(A) \cong$ $k \oplus k$ if $n=2 t$, and $H_{\mathrm{m}}^{t}(A) \cong H_{\mathrm{m}}^{n-t}(A) \cong k$ if $n \neq 2 t$.

Proof. It is obvious that $G$ satisfies the conditions of Theorem 2.1 with the element $y$ having the coordinates $y_{1}=y_{t+1}$ and $y_{i}=-1$ for $i \neq 1, t+1$. Hence $A$ is a quasi-Gorenstein ring. We have also proven in the proof of Theorem 2.1 that $S$ is a standard affine semigroup with $G(S)=G$ and

$$
S_{i}= \begin{cases}\left\{x \in G \mid x_{i} \geq 0 \text { and } x_{i} \neq 1\right\} & \text { if } i=1, t+1, \\ \left\{x \in G \mid x_{i} \geq 0\right\} & \text { if } i \neq 1, t+1 .\end{cases}
$$

From this it follows that $\operatorname{dim} A=\operatorname{rank}_{\mathbb{Z}} G=n-1$ and $S=\bigcap_{i=1}^{n} S_{i}$. Hence we can use Lemma 1.2 to compute the local cohomology modules $H_{\mathfrak{m}}^{i}(A)=$ $H_{M}^{i}(k[S]), i=1, \ldots, n-2$. First the form of the linear equation implies that

$$
\pi_{[1, n]}=\{J \subset[1, n] \mid J \nsupseteq[1, t],[t+1, n]\},
$$

where $[1, t]$ and $[t+1, n]$ denote the sets of the integers $1, \ldots, t$ and $t+1, \ldots, n$. For $J=[1, t], \pi_{J}$ is the simplicial complex of all proper subsets of $J$. The geometric realization $\left|\pi_{J}\right|$ of $\pi_{J}$ is homeomorphic to a $(t-2)$-dimensional sphere. Hence

$$
\widetilde{H}_{i-2}\left(\pi_{J} ; k\right)= \begin{cases}0 & \text { if } i \neq t, \\ k & \text { if } i=t .\end{cases}
$$

Similarly, for $J=[t+1, n]$, we have

$$
\widetilde{H}_{i-2}\left(\pi_{J} ; k\right)= \begin{cases}0 & \text { if } i \neq n-t \\ k & \text { if } i=n-t .\end{cases}
$$

If $|J| \leq n-2$ and $[1, t]$ is a proper subset of $J$, there exist integers $u, v \in$ $[t+1, n], u \in J, v \notin J$, and we can find solutions $x$ of the linear equation with $x_{i}=0$ for all $i \neq u, v, x_{u}<0$, and $x_{v}$ sufficiently large. Such solutions belong to $G_{J}$. Hence the supremum of the components of the elements of $G_{J}$ is infinite. By [11, Lemma 4.5], this implies that the simplicial complex $\pi_{J}$ is acyclic, i.e., all the reduced homology groups of $\pi_{J}$ vanish. Taking all these facts into consideration we obtain $H_{M}^{i}(k[S])=0$ for $i \neq t, n-t$ and

$$
H_{M}^{t}(k[S]) \cong k\left[G_{[1, t]}\right] \oplus k\left[G_{[t+1, n]}\right]
$$

if $t=n-t$, or

$$
\begin{aligned}
H_{M}^{t}(k[S]) & \cong k\left[G_{[1, t]}\right], \\
H_{M}^{n-t}(k[S]) & \cong k\left[G_{[t+1, n]}\right]
\end{aligned}
$$

if $t \neq n-t$. Now we are going to compute $G_{[1, t]}$. Let $x \in G_{[1, t]}$ be arbitrary. By the definition of the sets of $G_{J}$, we have $x \in \bigcap_{i=t+1}^{n} S_{i}$. Hence $x_{i} \geq 0$ for $i=t+1, \ldots, n$ and, therefore,

$$
(n-t) x_{t+1}+x_{t+2}+\cdots+x_{n} \geq 0 .
$$


On the other hand, since $x \notin S_{i}$ for $i=1, \ldots, t$, from the formula for $S_{i}$ we see that

$$
t x_{1}+x_{2}+\cdots+x_{t-1} \leq 0 \text {. }
$$

Hence both sides of the equation must be zero at $x$. But this happens only if $x_{1}=1, x_{i}=-1$ for $i=2, \ldots, t-1$, and $x_{i}=0$ for $i=t+1, \ldots, n$. That means $G_{[1, t]}$ consists of only one element $x$ with these components. Similarly, we show that $G_{[t+1, n]}$ consists of only one element $y$ with $y_{i}=0$ for $i=1, \ldots, t, y_{t+1}=1$, and $y_{i}=-1$ for $i=t+2, \ldots, n$. So we obtain the asserted formulas for the local cohomology modules of $A$. Moreover, since

$$
\bigcup_{i=1}^{n-2}\left\{x \in \mathbb{Z}^{n} \mid\left[H_{M}^{i}(k[S])\right]_{x} \neq 0\right\}=\{x, y\}
$$

and since $x-y$ and $y-x$ do not belong to $S$, the assumption of Lemma 1.3 is satisfied. Hence $A$ is a Buchsbaum ring.

Example. The affine semigroup $S$ of the preceding example yields a Buchsbaum quasi-Gorenstein ring $A$ with $\operatorname{dim} A=3$, $\operatorname{depth} A=2$, and $H_{\mathrm{m}}^{2}(A) \cong k \oplus k$.

Remark. Schenzel has shown that if $R$ is the graded ring $\bigoplus_{n \in \mathbb{N}} H^{0}\left(X, L^{\otimes n}\right)$, where $L$ is a very ample invertible sheaf of an abelian variety $X$ with $\operatorname{dim} X=$ $g>0$ and if $M=\bigoplus_{n>0} H^{0}\left(X, L^{\otimes n}\right)$, then $R_{M}$ is a Buchsbaum quasiGorenstein ring with $\operatorname{dim} R_{M}=g+1$, depth $R_{M}=2$, and

$$
\operatorname{dim}_{k} H_{M}^{i}\left(R_{M}\right)=\left(\begin{array}{c}
g \\
i-1
\end{array}\right)
$$

for $i=2, \ldots, g[8$, Theorem (4.1)].

According to [9], a prime quasi-Gorenstein ideal $P$ of a regular local ring $(R, M)$ is always linked to an almost complete intersection $Q$ of $R$. By generic link [5, Proposition 2.6], if we replace $R$ by a local ring of the form $S_{M S}$, where $S$ is a polynomial ring over $R$, we may assume that $Q$ is a prime ideal. Moreover, one can also show that

$$
H_{M}^{i-1}(R / Q) \cong H_{M}^{i}(R / P)
$$

for all $i<d=\operatorname{dim} R / Q$ and $H_{M}^{d-1}(R / Q)=0$ (see the proof of [9, Proposition $2]$ ). As the Buchsbaum property is preserved by linkage [1], we conclude that a Buchsbaum almost complete intersection local domain $R / Q$ is either a CohenMacaulay ring or $1 \leq \operatorname{depth} R / Q \leq(d-1) / 2$. The existence of Buchsbaum almost complete intersection local domains of any admissible depth follows from Lemma 2.2 by the theory of linkages.

Corollary 2.3. There exist Buchsbaum almost complete intersection local rings $A$ with $\operatorname{dim} A=d$ and $\operatorname{depth} A=t$ for any integers $d, t$ with $1 \leq t \leq(d-1) / 2$.

\section{REFERENCES}

1. H. Bresinsky, P. Schenzel, and W. Vogel, Liaison, arithmetical Buchsbaum curves, and monomial curves in $P^{3}$, J. Algebra 86 (1984), 283-301.

2. S. Goto and K. Watanabe, On graded rings. II: $\mathbb{Z}^{n}$-graded rings, Tokyo J. Math. 1 (1978), 237-261.

3. J. Herzog and E. Kunz, Die Wertehalbgruppe eines lokalen Rings der Dimension 1, Ber. Heidelberger Akad. Wiss., vol. II, 1971. 
4. M. Hochster, Rings of invariants of tori, Cohen-Macaulay rings generated by monomials, and polytopes, Ann. of Math. (2) 96 (1972), 318-337.

5. C. Huneke and B. Ulrich, Divisor class groups and deformations, Amer. J. Math. 107 (1985), 1265-1303.

6. C. Peskine and L. Szpiro, Liaison des variétés algébriques. I, Invent. Math. 26 (1974), 271302.

7. U. Schäfer and P. Schenzel, Dualizing complexes of affine semigroup rings, Trans. Amer. Math. Soc. 322 (1990), 561-582.

8. P. Schenzel, On Buchsbaum rings and their canonical modules, Seminar Eisenbud-SinghVogel, Vol. 1, Teubner-Texte Math., vol. 29, Teubner, Leipzig, 1980, pp. 65-76.

9. __ A note on almost complete intersections, Seminar Eisenbud-Singh-Vogel, Vol. 2, Teubner-Texte Math., vol. 48, Teubner, Leipzig, 1982, pp. 49-54.

10. R. Stanley, Hilbert functions of graded algebras, Adv. Math. 28 (1978), 57-83.

11. N. V. Trung and L. T. Hoa, Affine semigroups and Cohen-Macaulay rings generated by monomials, Trans. Amer. Math. Soc. 298 (1986), 145-167.

Mathematisches Institut, Universität Köln, 5000 Köln 41, Germany

E-mail address:mi059@mi.uni-koeln.de

Institute of Mathematics, Box 631, Bò Hô, Hanoi, Vietnam 